\title{
Laparoscopic personalized function-preserving gastrectomy with sentinel node mapping for early- stage gastric cancer
}

\author{
Hiroya Takeuchi ${ }^{1,2}$, Yuko Kitagawa ${ }^{2}$ \\ 1Department of Surgery, Hamamatsu University School of Medicine, Shizuoka 431-3192, Japan. \\ ${ }^{2}$ Department of Surgery, Keio University School of Medicine, Tokyo 160-8582, Japan.
}

Correspondence to: Dr. Hiroya Takeuchi, Department of Surgery, Hamamatsu University School of Medicine, 1-20-1 Handayama, Higashi-ku, Hamamatsu-shi, Shizuoka 431-3192, Japan. E-mail: takeuchi@hama-med.ac.jp

How to cite this article: Takeuchi H, Kitagawa Y. Laparoscopic personalized function-preserving gastrectomy with sentinel node mapping for early-stage gastric cancer. J Cancer Metastasis Treat 2018;4:38. http://dx.doi.org/10.20517/2394-4722.2017.83

Received: 12 Dec 2017 First Decision: 5 Feb 2018 Revised: 3 Jul 2018 Accepted: 9 Jul 2018 Published: 23 Jul 2018

Science Editor: Masayuki Watanabe Copy Editor: Jun-Yao Li Production Editor: Cai-Hong Wang

\begin{abstract}
Laparoscopic gastrectomy is considered as an indispensable option between endoscopic resection and standard gastrectomy with open laparotomy for patients with early-stage gastric cancer. However, the extent of gastrectomy and remnant gastric function may affect patients' quality of life (QOL) after surgery. Therefore, function-preserving gastrectomy in addition to laparoscopic surgery could be considered in patients with early-stage gastric cancer. A prospective multicenter trial and meta-analyses of sentinel node (SN) mapping and biopsy for early-stage gastric cancer have demonstrated favorable $\mathrm{SN}$ detection rates and accuracy of nodal metastatic status. Although a combination of radioactive colloids with blue dyes as tracers is currently considered as the promising procedure of SN mapping in earlystage gastric cancer, several new technologies, such as indocyanine green fluorescence imaging, may markedly improve its accuracy. For early-stage gastric cancer, the development of laparoscopic personalized minimized gastrectomy with SN mapping may help retain patents' QOL after surgery. A recently developed full-thickness partial gastrectomy with $\mathrm{SN}$ mapping and basin dissection would become a reliable minimally invasive gastrectomy for treating patients with cNO early-stage gastric cancer.
\end{abstract}

Keywords: Sentinel node, gastric cancer, laparoscopic, nonexposed endoscopic wall-inversion surgery

\section{INTRODUCTION}

In East Asian countries, such as Japan and Korea, early-stage gastric cancer (cT1) is identified in many patients owing to endoscopic diagnosis and surveillance ${ }^{[1]}$. Nowadays endoscopic submucosal dissection

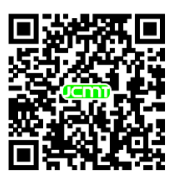


(ESD) is accepted as a less invasive procedure without gastrectomy for the resection of cT1 gastric cancer ${ }^{[1]}$. Laparoscopic gastrectomy is considered as an indispensable option between ESD and distal or total gastrectomy with open laparotomy for early-stage gastric cancer ${ }^{[2]}$. Laparoscopic distal gastrectomy (LDG) is comparable with open distal gastrectomy for early gastric cancer, and can be performed in clinical practice ${ }^{[3,4]}$. Many patients with gastric cancer currently undergo LDG and laparoscopic total gastrectomy (LTG) with standard lymphadenectomy ${ }^{[1-4]}$. LDG and LTG contribute to better aesthetics and earlier postoperative recovery after surgery ${ }^{\left[{ }^{[}\right]}$. However, the extent of gastrectomy and remnant gastric function may affect patients' quality of life (QOL) after surgery, resulting in several complications such as dumping syndrome and loss of body weight due to the disturbance of oral food intake. Therefore, function-preserving gastrectomy in addition to laparoscopic surgery could be considered in patients with early-stage gastric cancer indicated for these procedures.

Function-preserving minimized gastrectomy procedures, including partial and segmental gastrectomy, with modified lymphadenectomy are thought to improve postoperative gastric function compared to the standard gastrectomy. However, certain incidences of nodal skip metastasis in the second compartment or unpredicted station remain to be solved in these procedures. The sentinel node (SN) mapping and biopsy could overcome these issues as a novel intraoperative examination for accurate diagnosis of nodal metastasis in early-stage gastric cancer.

The SN is considered as the first lymph node(s) receiving lymphatic drainage from the primary tumor site ${ }^{[6,7]}$, and are regarded to be the first possible node(s) of metastasis from the primary lesion. Theoretically if SNs are pathologically negative for cancer metastasis, unnecessary extended lymphadenectomy can be avoided. $\mathrm{SN}$ navigation surgery is defined as a less invasive surgical procedure with modified lymphadenectomy by the diagnosis of SN metastasis. SN navigation surgery can prevent unnecessary lymphadenectomy and the occurrence of associated postoperative complications, and result in improving the patients' QOL.

SN mapping and biopsy were firstly utilized in breast cancer and melanoma, and subsequently attempted to other solid tumors ${ }^{[7-9]}$. Several studies involving SN mapping and biopsy for early-stage gastric cancer showed favorable SN detection rates and accuracy to predict nodal metastatic status ${ }^{[10,11]}$. Based on the studies, we have been developing a novel approach which combines laparoscopic function-preserving gastrectomy with SN mapping.

\section{LAPAROSCOPIC SN MAPPING AND BIOPSY PROCEDURES FOR GASTRIC CANCER}

Combination of radioactive colloids with blue or green dyes as a dual tracer method is currently thought to be the standard procedure for successful SN mapping in early-stage gastric cancer ${ }^{[10,11]}$. The accumulation of radioactive colloids in SN enables the detection of SN using hand-held gamma probes. In addition, blue dye is useful for real-time visualization of lymphatic flow even in laparoscopic surgery. Technetium ${ }^{99 m}$ tin colloids and technetium ${ }^{99 \mathrm{~m}}$ sulfur colloids are mainly utilized as radioactive tracers, and indocyanine green (ICG) is commonly used for dye tracer.

In our institutions, the indication to SN mapping and biopsy is currently limited to the patients with clinical T1 tumors over the ESD criteria, primary tumors of $<4 \mathrm{~cm}$ in tumor diameter, with clinical No gastric cancer $^{[10,11]}$. In our institution, $2.0 \mathrm{~mL}(150 \mathrm{MBq})$ of technetium ${ }^{99 \mathrm{~m}}$ tin colloid is injected endoscopically a day before surgery into the submucosal layer surrounding the primary lesion. Injection of the tracer into the submucosal layer using an endoscopic puncture needle facilitates more accurate tracer administration than laparoscopic injections from the seromuscular site of the gastric wall. Technetium ${ }^{99 m}$ tin colloid which has relatively large particle size (approximately $200 \mathrm{~nm}$ in diameter) accumulates in the SNs after the endoscopic injection into the primary tumor site. 
Blue or green dyes are also injected in the submucosal layer at primary lesion in a similar manner right after surgery began. Blue- or green-stained lymphatic vessels as well as lymph nodes are visualized during laparoscopic observation within $15 \mathrm{~min}$ after the injection. A hand-held gamma detector is also useful to locate the radioactive SN accurately. Moreover, laparoscopic gamma probing is feasible using a gamma detector which is available via trocar ports ${ }^{[10,11]}$.

For intraoperative SN biopsy, the pickup method is commonly employed in breast cancer and melanoma. However, the intraoperative SN sampling for gastric cancer should be accompanied with sentinel lymphatic basin dissection, which is a selected lymphatic basin dissection including identified $\mathrm{SN}^{[10,11]}$. The lymphatic basins around the stomach are currently divided into five basins along the main gastric arteries: basins along left gastric artery, right gastric artery, left gastroepiploic artery, right gastroepiploic artery, and posterior gastric artery ${ }^{[12]}$.

ICG has excitation and fluorescence wavelengths in the near-infrared range ${ }^{[13]}$. Many studies have clearly demonstrated the clinical utility of intraoperative ICG infrared imaging for laparoscopic SN mapping using infrared ray electronic endoscopy (IREE) to date ${ }^{[13,14]}$. IREE is useful to visualize ICG-stained lymphatic vessels and SN more clearly than normal laparoscopy. Subsequently, ICG fluorescence imaging was also developed as a reliable novel technique for SN mapping ${ }^{[15,16]}$. SN can be clearly visualized using laparoscopic ICG fluorescence imaging in comparison with conventional normal light imaging. Although the efficacy of ICG infrared or fluorescence imaging should be carefully evaluated by further prospective studies regarding SN detection rate and accuracy to predict the nodal metastasis, and compared with radio-guided methods, the new technologies may markedly improve the accuracy of laparoscopic SN mapping and biopsy in earlystage gastric cancer.

\section{FEASIBILITY OF SN MAPPING IN GASTRIC CANCER}

Until now, approximately 100 single institutional studies of SN mapping have indicated favorable SN detection rate and accuracy to predict nodal metastasis for early-stage gastric cancer. These results are as good as those of SN mapping for breast cancer and melanoma ${ }^{[11]}$. A meta-analysis, which consisted of 38 SN mapping studies including 2128 patients with gastric cancer, showed that the SN detection rate and accuracy of nodal status determination were $94 \%$ and $92 \%$, respectively ${ }^{[17]}$. The study also indicated that SN mapping for gastric cancer is reliable especially in patients with T1 tumor, use of dual tracers and submucosal injections of tracers.

A Japanese group previously conducted a prospective multicenter trial (UMIN ID: 000000476) to evaluate the feasibility of SN mapping for gastric cancer using the dual tracer method ${ }^{[10]}$. In this study, SN mapping and biopsy were performed for 397 patients with cT1NoMo or cT2NoMo single tumor with primary lesion diameter of $<4 \mathrm{~cm}$ and those without any previous treatment. To estimate the accuracy of the SN mapping, D2 or modified D2 gastrectomy was essentially performed for enrolled patients after SN mapping according to the guidelines for standard care by The Japan Gastric Cancer Association.

As the results of the study, the SN detection rate was $97.5 \%$ (387 of 397), and $14.7 \%$ of patients (57 of 387 ) showed lymph node metastasis. Fifty-three (93.0\%) of the 57 patients with nodal metastasis showed positive SN for metastasis. False-negative rate was $7 \%$ (4 of 57), and the overall accuracy to determine nodal metastatic status based on SN mapping was 99.0\% (383 of 387). Of the 53 patients with positive SN, 32 (60.4\%) had nodal metastases limited to only SN. Of the $21 \mathrm{SN}$-positive/non-SN-positive patients, 15 (71.4\%) had metastatic non-SN located within SN basins and 6 (28.6\%) had metastatic non-SN located outside the SN basins but within the extent of the D2 lymphadenectomy. Of the 4 patients with false-negative SN biopsy, 3 patients had either primary tumors of more than $4-\mathrm{cm}$ diameter or pT2 tumor or both, and only 1 patient who 
CT1 $(<4 \mathrm{~cm})$ NOM0 early gastric cancer

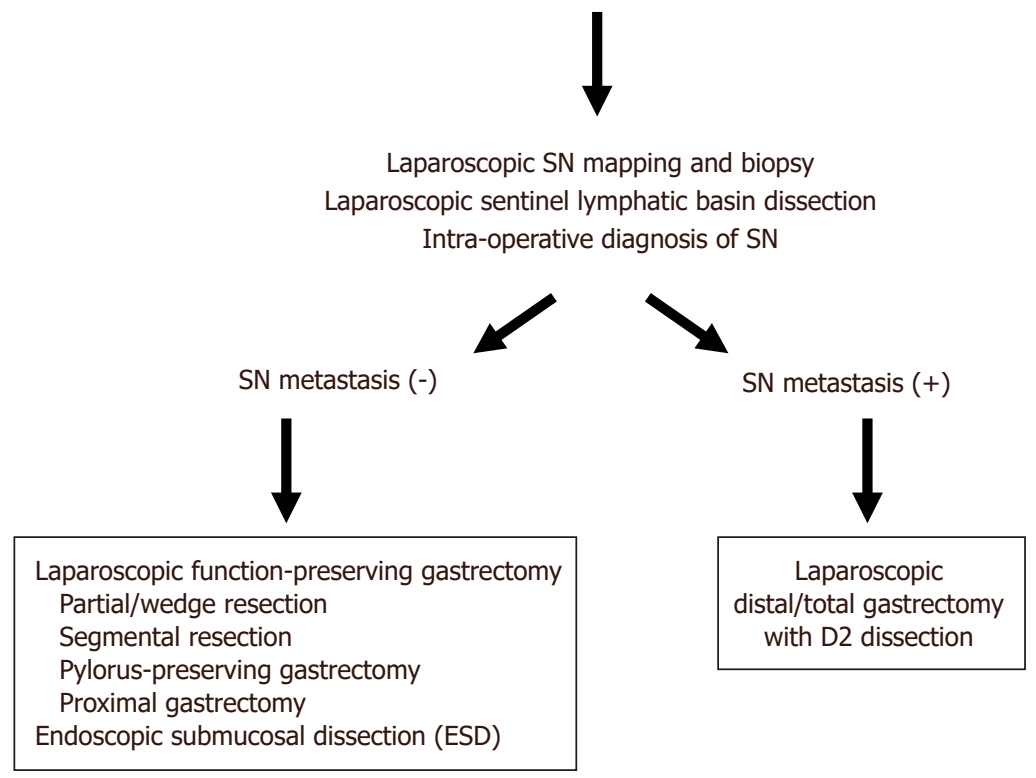

Figure 1. Laparoscopic function-preserving gastrectomy for CT1NOMO gastric cancer with sentinel node mapping. ESD: endoscopic submucosal dissection

had a primary tumor more than $4-\mathrm{cm}$ had a metastatic non-SN outside the $\mathrm{SN}$ basin ${ }^{[10]}$. The prospective multicenter trial verified that SN mapping for gastric cancer is technically feasible and reliable regarding SN detection rate and overall accuracy. The study would provide perspectives on the future of minimally invasive personalized gastrectomy based on SN mapping for early-stage gastric cancer.

\section{MINIMALLY INVASIVE GASTRECTOMY BASED ON SN MAPPING IN EARLY-STAGE GASTRIC CANCER}

Pathological status of SN and distribution of SN basins would provide the information in minimizing the extent of gastric resection and avoiding distal or total gastrectomy with D2 lymphadenectomy. Laparoscopic function-preserving gastrectomy for cT1No gastric cancers, including partial/wedge resection, segmental gastrectomy, proximal gastrectomy, and pylorus-preserving gastrectomy would be determined based on the SN status for each patient [Figure 1] ${ }^{[18-20]}$. Retention of patients' QOL in addition to earlier postoperative recovery could be obtained using laparoscopic minimized gastrectomy with SN mapping.

Ichikura et al. ${ }^{[21]}$ previously reported 35 patients with limited gastrectomy such as wedge resection and segmental gastrectomy with SN basin dissection for early gastric cancer with pathologically negative SN biopsy. As the results showed, all patients could survive without any recurrence of tumor in the study. Moreover the extent of the resected stomach in patients with limited gastrectomy was significantly less than that in patients with the standard gastrectomy. Based on these studies, our group in Japan has been conducting a multicenter prospective trial (UMIN ID: 000014401) which aims to elucidate laparoscopic function-preserving gastrectomy with SN mapping and SN basin dissection regarding long-term survival and postoperative patients' QOL for patients with clinical T1NoMo gastric cancer with primary lesions of $<4 \mathrm{~cm}$ in tumor diameter. In the study, en-bloc SN basin dissection including SN even in patients with SN-negative for metastasis is thought to be essential to warrant the curability of the surgery because of certain possibility of false-negative SN. A Korean group has also been conducting a prospective multicenter randomized controlled trial to clarify the oncological safety, including long-term survival, of laparoscopic functionpreserving gastrectomy with SN basin dissection compared with laparoscopic standard gastrectomy ${ }^{[22]}$. 

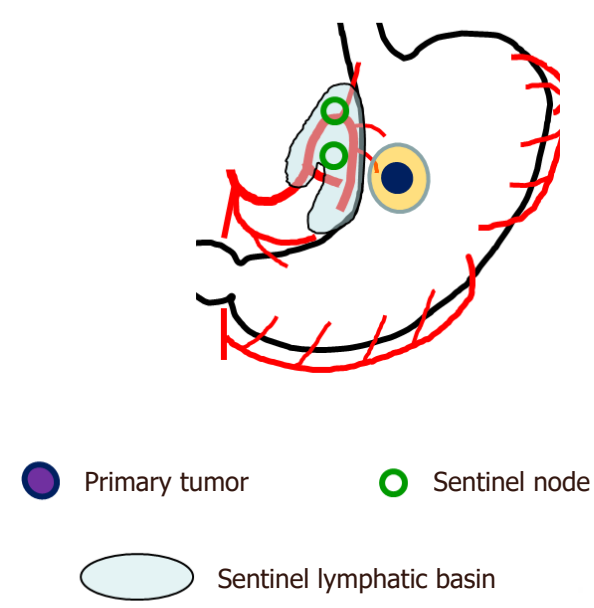

Non-exposed endoscopic wall-inversion surgery (NEWS)

Figure 2. Schema of nonexposed endoscopic wall-inversion surgery (NEWS) with laparoscopic sentinel node mapping and sentinel lymphatic basin dissection for early-stage gastric cancer

A combination of ESD with laparoscopic SN mapping for early-stage gastric cancer views another desirable option as a new minimally invasive stomach-preserving treatment. When all SNs are pathologically negative for metastasis in laparoscopic SN mapping and biopsy, then theoretically, ESD instead of gastrectomy might be sufficient for the curative resection of $\mathrm{CT} 1$ gastric cancer beyond the ESD criteria ${ }^{[20,23]}$. However, further studies are needed to certify the reliability of laparoscopic SN mapping with ESD.

Currently, LDG or LTG is frequently employed in patients with early-stage gastric cancer based on the pathological assessment of primary lesion obtained using ESD in the practice. Until now, whether SN mapping would be feasible or not even after ESD remains unknown. One of the most important concerns is that the lymphatic flow from the primary lesion to the original SN might be altered after ESD. However, a previous study reported that at least the SN basin was not markedly changed by ESD prior to surgery ${ }^{[20,23]}$. Laparoscopic limited gastrectomy based on SN mapping and biopsy could be feasible even after ESD.

\section{NONEXPOSED ENDOSCOPIC WALL-INVERSION SURGERY WITH MINIMALLY INVASIVE SN BIOPSY}

In laparoscopic partial gastrectomy, the demarcation line of the primary tumor cannot be identified accurately because the approach of gastrectomy is usually from the outside of the stomach. Therefore, a wider resection of the stomach cannot be avoided to prevent a positive surgical margin of primary tumor site. Recently, a new technique called nonexposed endoscopic wall-inversion surgery (NEWS) has been developed. The procedure is a full-thickness partial resection of the stomach, which can minimize the extent of gastric resection using endoscopic and laparoscopic surgeries without transluminal access designed to resect early-stage gastric cancer. In our ongoing clinical trial, the cases of NEWS with laparoscopic SN mapping and sentinel basin dissection have been accumulated in cT1NoMo early-stage gastric cancer [Figure 2] $]^{[24,25]}$.

In brief, after placing circumferential mucosal markings of primary tumor, ICG was endoscopically injected into the submucosal layer around the primary lesion to identify the SNs [Figure 3] $]^{[24]}$. The SN basin including SNs was dissected, and no metastasis in all SN was confirmed by intraoperative pathological examinations. After the SN mapping and biopsy, NEWS was performed for the resection 

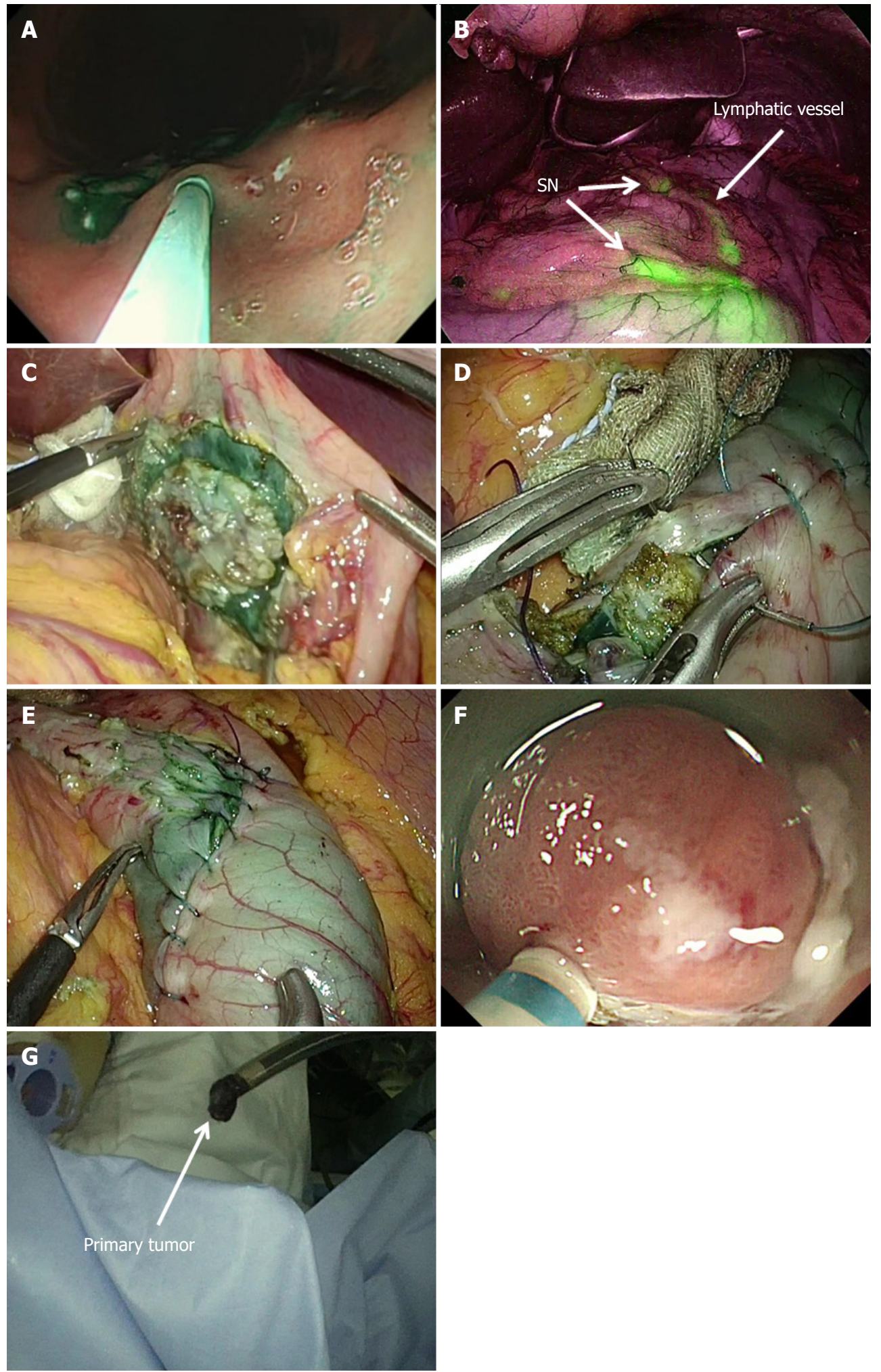

Figure 3. Nonexposed endoscopic wall-inversion surgery (NEWS) with laparoscopic sentinel node (SN) mapping and sentinel lymphatic basin dissection for early-stage gastric cancer. (A) Indocyanine green (ICG) was endoscopically injected into the submucosal layer around the primary lesion; (B) ICG florescence imaging results in clear visualization of SNs and lymphatics; (C) laparoscopic circumferential seromuscular incision surrounding the primary tumor; ( $D$ and $E$ ) laparoscopic seromuscular suturing and inversion of the primary tumor site to the inside of the stomach; (F) endoscopic circumferential mucosal and submucosal incision for primary tumor resection; (G) endoscopically retrieved primary tumor 
of primary tumor site. After placing circumferential serosal markings laparoscopically, submucosal injection was endoscopically administered. Next, circumferential seromuscular incision of the primary tumor and suturing of outer edge of the seromuscular incision were laparoscopically performed, with the primary lesion inverted to the inside of the stomach. Subsequently, the circumferential mucosal and submucosal incision of the primary lesion was endoscopically added, and the primary lesion was perorally removed [Figure 3].

NEWS in combination with laparoscopic SN mapping enables us to minimize the area of gastric resection as full-thickness partial gastrectomy in patients with SN-negative for metastasis ${ }^{[25]}$. NEWS does not require intentional perforation of the gastric wall during the procedure. Therefore we can apply this technique for treating gastric cancers without the risk of iatrogenic dissemination of tumor cells into the peritoneum and abdominal cavity. The NEWS combined with laparoscopic SN mapping are expected to become a promising minimally invasive, function-preserving gastrectomy to cure cNo early-stage gastric cancer.

\section{LIMITATION AND FUTURE PERSPECTIVE OF SN NAVIGATION SURGERY}

Many single institutional studies and the prospective multicenter trial of SN mapping and biopsy for early-stage gastric cancer have demonstrated acceptable SN detection rates and accuracy to predict nodal metastatic status. However, SN mapping techniques in details such as the choice of dyes, dual or single tracer methods, and the timing of tracer injection need to be standardized for universal application of SN biopsy in clinical practice. In addition, proper indication of laparoscopic function-preserving gastrectomy such as laparoscopic local resection with SN navigation surgery based on the tumor location has not been verified yet. Also, further improvement in SN navigation techniques should be required for more accurate SN mapping. Results of ongoing Japanese and Korean prospective trials would be expected to verify the postoperative patients' QOL and long-term survival in patients undergoing laparoscopic function-preserving gastrectomy with SN mapping.

\section{CONCLUSION}

For early-stage gastric cancer, good prognosis can currently be guaranteed by conventional standard gastrectomy. However, the personalized, minimally invasive treatments retaining the patients' QOL have to be developed as a next step. Although further studies are required for careful evaluation, laparoscopic functionpreserving gastrectomy, such as full-thickness partial gastrectomy, in combination with laparoscopic SN mapping would become an ideal strategy to reach the goal.

\section{DECLARATIONS}

\section{Authors' contributions}

Writing the manuscript: Takeuchi $\mathrm{H}$

Supervising as a primary investigator of the clinical trials in the manuscript: Kitagawa Y

\section{Availability of data and materials}

Not applicable.

\section{Financial support and sponsorship}

None.

\section{Conflicts of interest}

All authors declare that there are no conflicts of interest. 


\section{Ethical approval and consent to participate}

Not applicable.

\section{Consent for publication}

Not applicable.

\section{Copyright}

(c) The Author(s) 2018.

\section{REFERENCES}

1. Sano T, Hollowood K. Early gastric cancer: diagnosis and less invasive treatments. Scand J Surg 2006;95:249-55.

2. Kitano S, Iso Y, Moriyama M, Sugimachi K. Laparoscopy-assisted Billroth I gastrectomy. Surg Laparosc Endosc 1994;4:146-8.

3. Adachi Y, Shiraishi N, Shiromizu A, Shiromizu A, Bandoh T, Aramaki M, Kitano S. Laparoscopy-assisted Billroth I gastrectomy compared with conventional open gastrectomy. Arch Surg 2000;135:806-10.

4. Shinohara T, Kanaya S, Taniguchi K, Fujita T, Yanaga K, Uyama I. Laparoscopic total gastrectomy with D2 lymph node dissection for gastric cancer. Arch Surg 2009;144:1138-42.

5. Kim YW, Baik YH, Yun YH, Nam BH, Kim DH, Choi IJ, Bae JM. Improved quality of life outcomes after laparoscopy-assisted distal gastrectomy for early gastric cancer: results of a prospective randomized clinical trial. Ann Surg 2008;248:721-7.

6. Kitagawa Y, Fujii H, Mukai M, Kubota T, Ando N, Watanabe M, Ohgami M, Otani Y, Ozawa S, Hasegawa H, Furukawa T, Kumai K, Ikeda T, Nakahara T, Kubo A, Kitajima M. The role of the sentinel lymph node in gastrointestinal cancer. Surg Clin North Am 2000;80:1799-809.

7. Morton DL, Wen DR, Wong JH, Economou JS, Cagle LA, Storm FK, Foshag LJ, Cochran AJ. Technical details of intraoperative lymphatic mapping for early stage melanoma. Arch Surg 1992;127:392-9.

8. Giuliano AE, Kirgan DM, Guenther JM. Morton DL. Lymphatic mapping and sentinel lymphadenectomy for breast cancer. Ann Surg 1994;220:391-401.

9. Bilchik AJ, Saha S, Wiese D, Stonecypher JA, Wood TF, Sostrin S, Turner RR, Wang HJ, Morton DL, Hoon DS. Molecular staging of early colon cancer on the basis of sentinel node analysis: a multicenter phase II trial. J Clin Oncol 2001;19:1128-36.

10. Kitagawa Y, Takeuchi H, Takagi Y, Natsugoe S, Terashima M, Murakami N, Fujimura T, Tsujimoto H, Hayashi H, Yoshimizu N, Takagane A, Mohri Y, Nabshima K, Uenosono Y, Kinami S, Sakamoto J, Morita S, Aikou T, Miwa K, Kitajima M. Sentinel node mapping for gastric cancer: a prospective multicenter trial in Japan. J Clin Oncol 2013;31:3704-10.

11. Takeuchi H, Kitagawa Y. New sentinel node mapping technologies for early gastric cancer. Ann Surg Oncol 2013;20:522-32.

12. Kinami S, Fujimura T, Ojima E, Fushida S, Ojima T, Funaki H, Fujita H, Takamura H, Ninomiya I, Nishimura G, Kayahara M, Ohta T, Yoh Z. PTD classification: proposal for a new classification of gastric cancer location based on physiological lymphatic flow. Int J Clin Oncol 2008;13:320-9.

13. Tajima Y, Murakami M, Yamazaki K, Masuda Y, Kato M, Sato A, Goto S, Otsuka K, Kato T, Kusano M. Sentinel node mapping guided by indocyanine green fluorescence imaging during laparoscopic surgery in gastric cancer. Ann Surg Oncol 2010;17:1787-93.

14. Ishikawa K, Yasuda K, Shiromizu T, Etoh T, Shiraishi N, Kitano S. Laparoscopic sentinel node navigation achieved by infrared ray electronic endoscopy system in patients with gastric cancer. Surg Endosc 2007;21:1131-4.

15. Nimura H, Narimiya N, Mitsumori N, Yamazaki Y, Yanaga K, Urashima M. Infrared ray electronic endoscopy combined with indocyanine green injection for detection of sentinel nodes of patients with gastric cancer. Br J Surg 2004;91:575-9.

16. Miyashiro I, Miyoshi N, Hiratsuka M, Kishi K, Yamada T, Ohue M, Ohigashi H, Yano M, Ishikawa O, Imaoka S. Detection of sentinel node in gastric cancer surgery by indocyanine green fluorescence imaging: comparison with infrared imaging. Ann Surg Oncol 2008; $15: 1640-3$

17. Wang Z, Dong ZY, Chen JQ, Liu JL. Diagnostic value of sentinel lymph node biopsy in gastric cancer: a meta-analysis. Ann Surg Oncol 2012;19:1541-50.

18. Takeuchi H, Saikawa Y, Kitagawa Y. Laparoscopic sentinel node navigation surgery for early gastric cancer. Asian J Endosc Surg 2009;2:13-7.

19. Takeuchi H, Oyama T, Kamiya S, Nakamura R, Takahashi T, Wada N, Saikawa Y, Kitagawa Y. Laparoscopy-assisted proximal gastrectomy with sentinel node mapping for early gastric cancer. World J Surg 2011;35:2463-71.

20. Takeuchi H, Kitagawa Y. Sentinel node navigation surgery in patients with early gastric cancer. Dig Surg 2013;30:104-11.

21. Ichikura T, Sugasawa H, Sakamoto N, Yaguchi Y, Tsujimoto H, Ono S. Limited gastrectomy with dissection of sentinel node stations for early gastric cancer with negative sentinel node biopsy. Ann Surg 2009;249:942-7.

22. Park JY, Kim YW, Ryu KW, Nam BH, Lee YJ, Jeong SH, Park JH, Hur H, Han SU, Min JS, An JY, Hyung WJ, Cho GS, Jeong GA, Jeong O, Park YK, Jung MR, Yoon HM, Eom BW. Assessment of laparoscopic stomach preserving surgery with sentinel basin dissection versus standard gastrectomy with lymphadenectomy in early gastric cancer - a multicenter randomized phase III clinical trial (SENORITA trial) protocol. BMC Cancer 2016;16:340. 
23. Mayanagi S, Takeuchi H, Kamiya S, Niihara M, Nakamura R, Takahashi T, Wada N, Kawakubo H, Saikawa Y, Omori T, Nakahara T, Mukai M, Kitagawa Y. Suitability of sentinel node mapping as an index of metastasis in early gastric cancer following endoscopic resection. Ann Surg Oncol 2014;21:2987-93.

24. Goto O, Takeuchi H, Kawakubo H, Sasaki M, Matsuda T, Matsuda S, Kigasawa Y, Kadota Y, Fujimoto A, Ochiai Y, Horii J, Uraoka T, Kitagawa Y, Yahagi N. First case of non-exposed endoscopic wall-inversion surgery with sentinel node basin dissection for early gastric cancer. Gastric Cancer 2015;18:440-5.

25. Takeuchi H, Kitagawa Y. Sentinel lymph node biopsy in gastric cancer. Cancer J 2015;21:21-4. 